\title{
Computers
} \& Structures

\section{The inf-sup condition and its evaluation for mixed finite element methods}

\author{
Klaus-Jürgen Bathe * \\ Department of Mechanical Engineering, Massachusetts Institute of Technology, 77 Mass Avenue, Cambridge, MA 02139, USA
}

Accepted 16 November 1999

\begin{abstract}
The objective of this paper is to review the general inf-sup condition for mixed finite element methods and summarize numerical procedures (inf-sup tests) for the evaluation of the inf-sup expressions specific to various problem areas. The inf-sup testing of a given mixed finite element discretization is most important in order to assess its reliability and solution effectiveness. The problem areas considered are (almost) incompressible analysis of solids and fluids, acoustic fluids, the analysis of plates and shells, and the solution of convection-dominated flows. (C) 2000 Elsevier Science Ltd. All rights reserved.
\end{abstract}

\section{Introduction}

The finite element method is now abundantly used for the analysis of solids and structures, fluids and fluidstructure interactions. For the analysis of solids and structures, displacement-based finite element discretizations are most attractive; however, for some analyses, mixed methods need to be employed. For the analyses of fluid flows, mixed finite element procedures are generally used [1].

While - for the analysis of solids and structures - the displacement-based finite element procedures are formulated in a straightforward manner using the principle of virtual displacements, effective mixed methods are much more difficult to establish. These formulations must be employed for the analyses of (almost) incompressible response, such as those encountered in elastic solutions of rubber-like materials, and in inelastic solutions of many materials. Mixed formulations need also be employed for the analyses of plates and shells.

Considering fluid flows, it is natural to use a mixed formulation because the governing differential equations

\footnotetext{
${ }^{*}$ Tel.: +1-617-2536645; fax: +1-617-2532275.
}

already involve multiple field variables; for example, for incompressible fluid flow, the velocities and pressure are natural solution variables to use. For the analysis of fluid-structure interactions, therefore, mixed formulations are also widely used.

The first mixed formulations were proposed using suitable variational principles of the continuum problem, such as the $\mathrm{Hu}$-Washizu and Hellinger-Reissner principles. Reasonable finite element interpolations were chosen and numerical test cases were solved to demonstrate the effectiveness of the formulations. However, while for the displacement-based finite element method a few test cases can indeed show the overall effectiveness of a discretization scheme, a mixed method may work remarkably well in the solution of some problems and totally fail in other problems. The fact that the mixed finite element method is based on a proper variational formulation does not at all ensure that the finite element method is reliable and that the method can be recommended for general use (see p. 477 of Ref. [1]).

Considering a mixed finite element discretization, in whichever way the discretization has been formulated, it is crucial that the scheme be analyzed for its mathematical convergence characteristics [1,2]. The specific conditions to be fulfilled are the consistency, ellipticity, and inf-sup conditions. The consistency and ellipticity 
conditions are fulfilled relatively easily. However, the applicable inf-sup condition is frequently difficult to satisfy. Since an analytical proof to identify whether the inf-sup condition is satisfied is frequently out of reach, numerical inf-sup test procedures have been designed [1,3-6].

It is also possible to design a mixed method in which an inf-sup condition is by-passed [7,8]. However, in that case, in general, numerical (artificial) constants enter the formulation. The choice of these constants is a source of difficulty, even though a mathematical analysis might give guidelines as to the values to be used. It is more desirable to not introduce numerical constants in the formulation and design a method which does satisfy the applicable inf-sup condition.

Our experience is that if the applicable inf-sup condition is satisfied (and of course, the consistency and ellipticity requirements are fulfilled as well), the finite element scheme is reliable and effective and never "fails". This is the reason why we endeavor to only use finite element discretization schemes that satisfy the condition. However, the inf-sup condition is a severe requirement and a method not fulfilling the condition might still be acceptable.

The objective of this paper is to present some latest experiences in the use and numerical evaluation of the inf-sup condition in various analysis areas. In the following sections, we first present the inf-sup condition in two general forms, and then show how specific expressions are applied to finite element formulations for incompressible analysis, acoustic analysis, plate and shell structures and high Péclet and Reynolds number flows. In the presentation, we endeavor to keep the discussion short and hence do not give details of derivation but refer to appropriate references, where these details can be found. We conclude that mixed finite element formulations can now be evaluated as shown in this paper before it is claimed that an effective formulation has been reached.

A brief explanation regarding the notation used might be helpful. All continuous and finite element variables are defined in $\mathbb{R}^{N}, N=1,2$ or 3 depending on the problem considered and are always simply presented as italic symbols. In the definition of the functional spaces used, the given properties apply to each vector component. However, the finite element matrices and the nodal point solution vectors are given in bold roman symbols.

\section{The inf-sup condition in a general setting}

In this section we review the inf-sup condition in two different general forms. Applications are then presented in Section 3.
2.1. The inf-sup condition using the complete bilinear form

Consider a general variational problem given in a Hilbert space $\mathscr{U}$ with a bilinear (symmetric or nonsymmetric) form $A(\phi, \psi)$ defined on $\boldsymbol{U} \times \mathscr{U}$. The first argument in the bilinear form $A(\cdot, \cdot)$ is a solution function and the second argument is a weighting function. We define the following space:

$$
\begin{gathered}
\mathscr{U}=\left\{u \mid u \in L^{2}(\mathrm{Vol}) ; \frac{\partial u}{\partial x_{k}} \in L^{2}(\mathrm{Vol}), k=1,2,3 ;\right. \\
\left.u=0 \text { on } S_{u}\right\},
\end{gathered}
$$

where we assume zero Dirichlet boundary conditions on $S_{u}$ and $L^{2}(\mathrm{Vol})$ is the space of square integrable functions in the volume, "Vol", of the body considered,

$$
\begin{aligned}
& L^{2}(\mathrm{Vol})=\{u \mid u \text { is defined in } \mathrm{Vol} \text { and } \\
&\left.\int_{\mathrm{Vol}} u^{2} \mathrm{dVol}=\|u\|_{L^{2}(\mathrm{Vol})}^{2}<+\infty\right\} .
\end{aligned}
$$

Given a linear functional $(F, \psi)$ from $\mathscr{U}$ to $\mathbb{R}$, we have for the continuous problem:

Find $\phi \in \mathscr{U}$ such that

$A(\phi, \psi)=(F, \psi) \quad \forall \psi \in \mathscr{U}$,

where $F$ is the forcing term. For nonzero Dirichlet boundary conditions, the problem statement can of course also be reduced to Eq. (3) by including the effect of the boundary conditions in $(F, \psi)[1]$. We assume that there exists a unique solution of Eq. (3).

The finite dimensional subspace of $\mathscr{U}$ is defined as follows:

$$
\begin{gathered}
\mathscr{U}_{h}=\left\{u_{h} \mid u_{h} \in L^{2}(\mathrm{Vol}) ; \frac{\partial u_{h}}{\partial x_{k}} \in L^{2}(\mathrm{Vol}), k=1,2,3 ;\right. \\
\left.u_{h} \in T_{n}\left(\mathrm{Vol}^{(m)}\right) ; u_{h}=0 \text { on } S_{u}\right\}
\end{gathered}
$$

where $T_{n}\left(\mathrm{Vol}^{(m)}\right)$ denotes a finite element interpolation of order $n$ over the element $m$ of volume $\mathrm{Vol}^{(m)}$.

The finite element solution of Eq. (3) is obtained by solving the following finite dimensional problem:

Find $\phi_{h} \in \mathscr{U}_{h}$ such that

$$
A\left(\phi_{h}, \psi\right)=(F, \psi) \quad \forall \psi \in \mathscr{U}_{h} .
$$

To measure the quality of the finite element approximation, we need to introduce norms. Let $\|\cdot\|_{S}$ be the norm that we use to measure the size of the solution functions and $\|\cdot\|_{T}$ be the norm for the weighting functions. Then the continuity condition is

$$
A(\eta, \psi) \leqslant k_{A}\|\eta\|_{S}\|\psi\|_{T} \quad \forall \eta, \psi \in \mathscr{U},
$$


where $k_{A}$ is a (finite) constant. This equation, assumed satisfied, simply states that the bilinear form $A(\eta, \psi)$ behaves normally.

Then, the classical condition to be satisfied to have a stable and optimal procedure for the solution of Eq. (5) is the inf-sup condition:

Inf-sup condition on $A(\cdot, \cdot)$ :

$\exists$ a constant $\gamma>0$ and independent of crucial physical parameters and the element size $h$ such that

$\inf _{\eta \in \mathscr{U}_{h}} \sup _{\psi \in \mathscr{U}_{h}} \frac{A(\eta, \psi)}{\|\eta\|_{S}\|\psi\|_{T}} \geqslant \gamma$

This condition ensures the solvability, stability and optimality of the finite element solution.

The inf-sup condition is crucial in establishing the quality of the finite element approximation. In general, we have the following relation $[1,2]$ :

$\left\|\phi-\phi_{h}\right\|_{S} \leqslant\left(1+\frac{k_{A}}{\gamma}\right) \inf _{\eta_{h} \in \mathscr{U}_{h}}\left\|\phi-\eta_{h}\right\|_{S}$.

Let us show that the inf-sup condition implies the inequality (8).

From the inequality (7) with $\eta_{h}=\chi_{h}-\phi_{h}$, for any $\chi_{h} \in \mathscr{U}_{h}$ we have

$$
\begin{aligned}
\gamma\left\|\chi_{h}-\phi_{h}\right\|_{S} & \leqslant \sup _{\psi_{h} \in \mathscr{U}_{h}} \frac{A\left(\chi_{h}-\phi_{h}, \psi_{h}\right)}{\left\|\psi_{h}\right\|_{T}} \\
& =\sup _{\psi_{h} \in \mathscr{U}_{h}} \frac{A\left(\chi_{h}-\phi, \psi_{h}\right)+A\left(\phi-\phi_{h}, \psi_{h}\right)}{\left\|\psi_{h}\right\|_{T}} \\
& =\sup _{\psi_{h} \in \mathscr{U}_{h}} \frac{A\left(\chi_{h}-\phi, \psi_{h}\right)}{\left\|\psi_{h}\right\|_{T}} \\
& \leqslant \sup _{\psi_{h} \in \mathscr{U}_{h}} \frac{k_{A}\left\|\chi_{h}-\phi\right\|_{S}\left\|\psi_{h}\right\|_{T}}{\left\|\psi_{h}\right\|_{T}} \\
& =k_{A}\left\|\chi_{h}-\phi\right\|_{S},
\end{aligned}
$$

where we used Eq. (6) and that

$A\left(\phi-\phi_{h}, \psi_{h}\right)=0 \quad \forall \psi_{h} \in \mathscr{U}_{h}$.

Using the triangle inequality, we thus have

$$
\begin{aligned}
\left\|\phi_{h}-\phi\right\|_{S} & \leqslant\left\|\phi_{h}-\chi_{h}\right\|_{S}+\left\|\chi_{h}-\phi\right\|_{S} \\
& \leqslant \frac{k_{A}}{\gamma}\left\|\chi_{h}-\phi\right\|_{S}+\left\|\chi_{h}-\phi\right\|_{S} \\
& =\left(1+\frac{k_{A}}{\gamma}\right)\left\|\chi_{h}-\phi\right\|_{S},
\end{aligned}
$$

which proves the inequality (8).

We may note that we can write Eq. (8) as

$$
\left\|\phi_{h}-\phi\right\|_{S} \leqslant \beta d\left(\phi, \mathscr{U}_{h}\right),
$$

where $\beta$ is a constant, equal to $\left(1+k_{A} / \gamma\right)$, and $d\left(\phi, \mathscr{U}_{h}\right)$ is the distance between the exact solution $\phi$ and the finite element interpolation space $\boldsymbol{U}_{h}$, $d\left(\phi, \mathscr{U}_{h}\right)=\inf _{\eta_{h} \in \mathscr{U}_{h}}\left\|\phi-\eta_{h}\right\|_{S}$.

If the inf-sup condition is satisfied, with $\gamma$ not too small, and if $k_{A}$ is not too large, the constant $\left(1+k_{A} / \gamma\right)$ in Eq. (8) is well behaved. Interpolation theory is next used to obtain error bounds measured on the element size $h$. For example, consider the three-dimensional elasticity problem and let $\|\cdot\|_{S}$ be the usual $H^{1}$ Sobolev norm. Interpolation theory gives that for problems with sufficiently smooth solutions, for some $\eta_{h} \in \mathscr{U}_{h}$,

$\left\|\phi-\eta_{h}\right\|_{H^{1}} \leqslant c h^{k}$,

where $h$ denotes the characteristic element size, $k$ is the order of the complete polynomial in the finite elements used, and $c$ is a constant, which depends on the problem considered (the solution) but is independent of $h$. Combining the relations (8) and (14), we have the optimal error bound

$\left\|\phi-\phi_{h}\right\|_{H^{1}} \leqslant c\left(1+\frac{k_{A}}{\gamma}\right) h^{k} \cong c^{*} h^{k}$,

where $c^{*}$ is a constant which depends on the problem considered and is inversely proportional to $\gamma$. Since $c, k_{A}$ and $\gamma$ are independent of $h$, so is $c^{*}$.

The formulation that we considered in this section is of very general mixed form. We now proceed to consider a mixed formulation which is more specialized and is encountered widely in applications.

\subsection{The inf-sup condition for the constraint mixed formulation}

Consider now the general mixed formulation of a continuous problem in the classical form:

Given two Hilbert spaces $\mathscr{U}$ and $\mathscr{E}$, find $u \in \mathscr{U}$ and $\varepsilon \in \mathscr{E}$ such that

$$
\begin{aligned}
& a(u, v)+b(\varepsilon, v)=(f, v) \quad \forall v \in \mathscr{U}, \\
& b(\eta, u)-c(\varepsilon, \eta)=0 \quad \forall \eta \in \mathscr{E},
\end{aligned}
$$

where $a(\cdot, \cdot), b(\cdot, \cdot)$ and $c(\cdot, \cdot)$ are bilinear forms, with $a(\cdot, \cdot)$ and $c(\cdot, \cdot)$ symmetric forms, $(f, \cdot)$ is a linear form and, of course, $\mathscr{U}$ and $\mathscr{E}$ are the spaces corresponding to the problem considered. The second relation in Eq. (16) provides of course the constraint in the "mixed fields" of the formulation. As in the previous discussion, for simplicity of presentation, we assume that the prescribed boundary conditions on the solution variables are homogeneously zero. We also assume that

$$
\begin{aligned}
& a(v, w) \leqslant k_{a}\|v\|_{\mathscr{U}}\|w\|_{\mathscr{U}} \quad \forall v, w \in \mathscr{U}, \\
& b(q, v) \leqslant k_{b}\|v\|_{\mathscr{U}}\|q\|_{\mathscr{E}} \quad \forall v \in \mathscr{U}, q \in \mathscr{E},
\end{aligned}
$$

where we use $\|\cdot\|_{\mathscr{U}}$ and $\|\cdot\|_{\mathscr{E}}$ to measure the size of the functions in $\mathscr{U}$ and $\mathscr{E}$, and $k_{a}$ and $k_{b}$ are continuity constants, and that 
$c(q, q) \leqslant k_{c}\left\|q_{1}\right\|_{\mathscr{E}}\left\|q_{2}\right\|_{\mathscr{E}} \quad \forall q_{1}, q_{2} \in \mathscr{E}$,

$c(q, q) \geqslant 0 \quad \forall q \in \mathscr{E}$

with $k_{c}$ also a continuity constant. As in Section 2.1, we assume that the continuous problem has a unique solution.

The finite element solution of this problem is obtained by choosing finite-dimensional subspaces of $\mathscr{U}$ and $\mathscr{E}$, which we call $\mathscr{U}_{h}$ and $\mathscr{E}_{h}$, respectively. Then the finite element problem is:

Find $u_{h} \in \mathscr{U}_{h}$ and $\varepsilon_{h} \in \mathscr{E}_{h}$ such that

$a\left(u_{h}, v\right)+b\left(\varepsilon_{h}, v\right)=(f, v) \quad \forall v \in \mathscr{U}_{h}$,

$b\left(\eta, u_{h}\right)-c\left(\varepsilon_{h}, \eta\right)=0 \quad \forall \eta \in \mathscr{E}_{h}$.

This mixed formulation should satisfy the following two classical conditions to have an optimal procedure:

(i) Ellipticity of $a(\cdot, \cdot)$ on the null-space of $b(\cdot, \cdot)$ :

$\exists$ a constant $\alpha>0$ such that

$a(v, v) \geqslant \alpha\|v\|_{\mathscr{U}}^{2} \quad \forall v \in \mathscr{U}_{0}^{h}$,

where

$\boldsymbol{U}_{0}^{h}=\left\{v \in \mathscr{U}_{h} \mid b(\psi, v)=0 \quad \forall \psi \in \mathscr{E}_{h}\right\}$

is the null space of $b(\cdot, \cdot)$. Here we assume that $c(\cdot, \cdot)$ can be zero, see the relations (18).

(ii) Inf-sup condition on $b(\cdot, \cdot)$ :

$\exists$ a constant $\gamma>0$ independent of crucial physical parameters and the element size $h$, such that

$\sup _{v \in \mathscr{U}_{h}} \frac{b(\eta, v)}{\|v\|_{\mathscr{U}}} \geqslant \gamma \sup _{v \in \mathscr{U}} \frac{b(\eta, v)}{\|v\|_{\mathscr{U}}} \quad \forall \eta \in \mathscr{E}_{h}$.

This inf-sup condition follows from the Banach theorems [9] and corresponds to a general form. In specific formulations, we seek to rewrite the supremum expression on the right-hand side to obtain a more tractable form (see Sections 3.1-3.4).

When the inf-sup condition holds (and the ellipticity condition is satisfied), the following relation can be established $[1,2]$ :

$\left\|u-u_{h}\right\|_{\mathscr{U}}+\left\|\varepsilon-\varepsilon_{h}\right\|_{\mathscr{E}} \leqslant c \inf _{v \in \mathscr{U}_{h}, \eta \in \mathscr{E}_{h}}\left(\|u-v\|_{\mathscr{U}}+\|\varepsilon-\eta\|_{\mathscr{E}}\right)$

with $c$ a constant which depends on $k_{a}, k_{b}, k_{c}, \alpha$ and $\gamma$ (and not $h$ ).

Assuming that the solution is sufficiently smooth and using interpolation theory, the optimal error bounds are obtained

$\left\|u-u_{h}\right\|_{\mathscr{U}}+\left\|\varepsilon-\varepsilon_{h}\right\|_{\mathscr{E}} \cong c^{*} h^{k}$,

where $k$ is the complete polynomial order used in the interpolation space $\mathscr{U}_{h}$, the interpolations in the space $\mathscr{E}_{h}$ are performed with appropriate complementary orders, and $c^{*}$ is a (new) constant which depends on the constant $c$ in Eq. (23) and hence on $\gamma$ (actually on $\gamma^{-1}$ ) and also on the problem considered (the solution) but is independent of the element size $h$. Note that Eq. (23) is like Eq. (8) and Eq. (24) is like Eq. (15).

Hence, as in Section 2.1, it is crucial that the inf-sup condition be satisfied in order to have an effective finite element procedure.

\section{Applications of the inf-sup condition}

The objective of this section is to show how the infsup conditions in Eqs. (7) and (22) are applied and evaluated in various analysis fields. In some cases analytical evaluations have been achieved (see, for example, Ref. [1]) but we focus here on those finite element formulations, where an analytical evaluation has been out of reach. In that case, a numerical evaluation is very valuable, and while not as encompassing as an analytical proof, our experience is that when the numerical test is passed, in fact, the inf-sup condition is satisfied. A numerical test is like the patch test (used for nonconforming displacement discretizations) usually performed numerically instead of analytically.

\subsection{Incompressible elasticity (and Stokes flow)}

For the solution of an incompressible (or almost incompressible) elasticity problem, the displacement/pressure $(u / p)$ finite element discretizations are effective [1]. Let $V_{h}$ be the finite element displacement interpolation space and $Q_{h}$ be the finite element pressure interpolation space (corresponding to the spaces $V$ and $Q$ of the continuous problem). Then the finite element problem is:

Find $u_{h} \in V_{h}$ and $p_{h} \in Q_{h}$ such that

$$
\begin{aligned}
a^{\mathrm{IE}}\left(u_{h}, v_{h}\right)+b\left(p_{h}, v_{h}\right) & =\left(f, v_{h}\right) \quad \forall v_{h} \in V_{h}, \\
b\left(q_{h}, u_{h}\right)-\frac{1}{\kappa}\left(p_{h}, q_{h}\right) & =0 \quad \forall q_{h} \in Q_{h},
\end{aligned}
$$

where $\kappa$ is the bulk modulus,

$b\left(q_{h}, v_{h}\right)=\int_{\mathrm{Vol}} q_{h} \operatorname{div} v_{h} \mathrm{dVol}$

and $a^{\mathrm{IE}}(\cdot, \cdot)$ is the bilinear form of the deviatoric strain energy.

Considering the presentation in Section 2.2, the norms used here are $\|\cdot\|_{\mathscr{U}} \equiv\|\cdot\|_{H^{1}}$ and $\|\cdot\|_{\mathscr{E}} \equiv\|\cdot\|_{L^{2}}$. The inf-sup condition is in this case obtained from Eq. (22) by bounding the supremum on the right-hand side as

$c_{1}\left\|q_{h}\right\|_{L^{2}} \geqslant \sup _{v \in V} \frac{\int_{\mathrm{Vol}} q_{h} \operatorname{div} v \mathrm{dVol}}{\|v\|_{H^{1}}} \geqslant c_{2}\left\|q_{h}\right\|_{L^{2}}$

with $c_{1}$ and $c_{2}$ constants. 
Hence, the $L^{2}$-norm of $q_{h}$ is equivalent to the righthand side of Eq. (22) and the inf-sup condition is for this problem

$$
\inf _{q_{h} \in Q_{h} v_{h} \in V_{h}} \frac{\int_{\mathrm{Vol}} q_{h} \operatorname{div} v_{h} \mathrm{dVol}}{\left\|v_{h}\right\|_{H^{1}}\left\|q_{h}\right\|_{L^{2}}}=\gamma_{h} \geqslant \gamma>0
$$

with $\gamma$ a constant independent of $h$. Note that we consider here the almost incompressible case and the fully incompressible (the limit) case. This inf-sup condition is frequently referred to as the Babuška-Brezzi (or simply $\mathrm{BB})$ condition because of the seminal papers by Babuška [10] and Brezzi [11]. The condition is also referred to as the LBB condition including the name of Ladyzhenskaya [12]. Various $u / p$ finite element discretization schemes have been proven analytically to satisfy this condition (see, for example Ref. [1]). However, some element discretizations and discretizations based on distorted element meshes (used virtually always in engineering practice) could not (yet) be proven analytically to satisfy the condition, and therefore a numerical test was proposed by Chapelle and Bathe [3].

For the inf-sup test, the matrices $\boldsymbol{G}_{h}$ and $\boldsymbol{S}_{h}$ are defined for a given finite element discretization. The mesh used for a given geometry, including the displacement boundary conditions, gives

$$
\int_{\mathrm{Vol}} q_{h} \operatorname{div} v_{h} \mathrm{dVol}=\boldsymbol{W}_{h}^{\mathrm{T}} \boldsymbol{G}_{h} \boldsymbol{V}_{h}
$$

$\left\|q_{h}\right\|_{L^{2}}^{2}=\boldsymbol{W}_{h}^{\mathrm{T}} \boldsymbol{G}_{h} \boldsymbol{W}_{h}$,

$\left\|v_{h}\right\|_{H^{1}}^{2}=\boldsymbol{V}_{h}^{\mathrm{T}} \boldsymbol{S}_{h} \boldsymbol{V}_{h}$,

where $\boldsymbol{W}_{h}$ and $\boldsymbol{V}_{h}$ are the finite element nodal point solution vectors. In practice, the semi-norms instead of the full norms are used (based on the PoincaréFriedrichs inequality [1]).

The smallest nonzero eigenvalue $\lambda_{k}$ of the following problem is then calculated to obtain the inf-sup value $\gamma_{h}$, as detailed in Ref. [1],

$\boldsymbol{G}_{h} \boldsymbol{\phi}=\lambda \boldsymbol{S}_{h} \boldsymbol{\phi}$.

The value $\sqrt{\lambda_{k}}$ is equal to the inf-sup value $\gamma_{h}$ in Eq. (28) for the discretization considered provided that there are no spurious pressure modes. The number of spurious pressure modes can be predicted from

$k_{\mathrm{pm}}=k-\left(n_{u}-n_{p}+1\right)$,

where $n_{u}$ is the number of displacement degrees of freedom and $n_{p}$ is the number of pressure degrees of freedom. If $k_{\mathrm{pm}}>0$, the finite element discretization contains the constant pressure mode or spurious pressure modes [1]. The physical constant pressure mode can be eliminated by changing the boundary conditions. If a spurious pressure mode is present, $\gamma_{h}$ is zero and the inf-

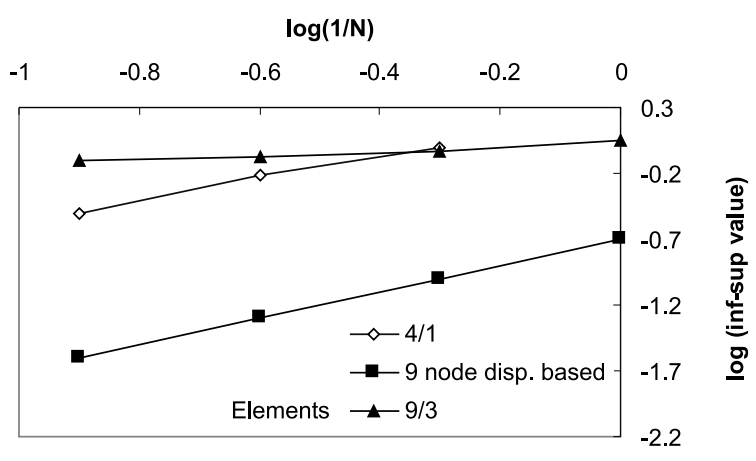

Fig. 1. Inf-sup test results for the (almost or fully) incompressible analysis case. A square cantilever plate using meshes of $N$ by $N$ elements is considered.

sup test is of course not passed (the inf-sup condition is not satisfied).

The test involves the calculation of $\gamma_{h}$ for a sequence of meshes with increasingly finer discretizations; typically meshes are used with element sizes $h, h / 2$, $h / 4, h / 8, \ldots$ If the inf-sup values for these discretizations do not show a decrease towards zero, the test is passed (provided that there are also no spurious pressure modes). Hence, the element is optimal as discussed in Section 2.2. Since the constant " $\gamma$ " in Eq. (28) does not depend on $h$ (and of course not on the bulk modulus which approaches infinity as Poisson's ratio approaches 0.5 ), the element does not "lock".

Fig. 1 shows results reported in Ref. [3] for the testing of various elements. Note that any loading and material properties do not enter the test. The boundary conditions, on the other hand, do enter the test and hence a judiciously chosen problem should be considered.

Note that this test also shows whether an element is optimal in almost incompressible conditions such as encountered in inelastic analysis. Indeed, only elements that pass this test can be recommended for the solution of almost or fully incompressible media, including, in particular, also elasto-plastic conditions [1].

The definition of $\boldsymbol{G}_{h}$ in Eq. (30) is for the $u / p$ formulation in which inter-element discontinuous pressure interpolations are used. In case inter-element continuous pressure interpolations are employed (referred to as the $u / p-c$ formulation) the matrix $\boldsymbol{G}_{h}$ is different [1].

The test is also directly applicable to fluid flow elements used to solve Stokes flow by simply using the velocity instead of the displacement variables, and can of course also be employed for elements based on enhanced strain fields [13].

\subsection{Acoustic fluid}

If an acoustic fluid is modeled using the displacements and pressure as variables, the formulation discussed in 


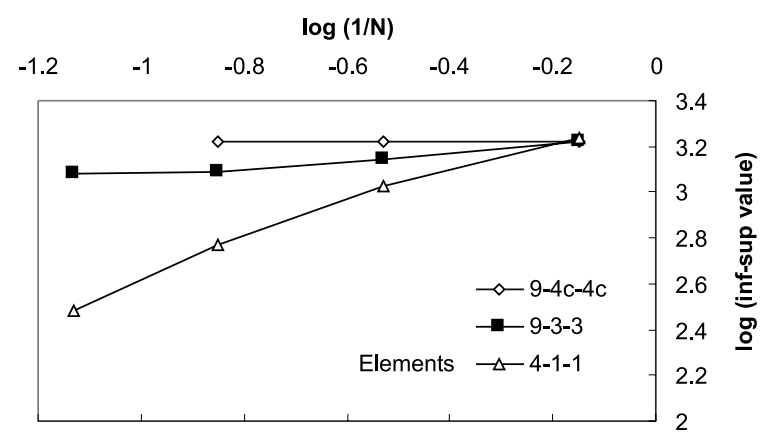

Fig. 2. Inf-sup test results for the irrotational acoustic fluid elements. Fluid in a rigid cavity using meshes of $N$ by $N$ elements is considered [15].

the previous section is obtained. In this case, the bilinear form $a^{\mathrm{IE}}(\cdot, \cdot)$ is zero (or of small magnitude) because the fluid is (almost) inviscid, and many physical zero (or small) frequencies are encountered. Some of these zero frequencies can be eliminated by imposing the constraint of zero vorticity using $[14,15]$

$\nabla \times u=\frac{\lambda}{\alpha}$,

where $\lambda$ is a vorticity moment and $\alpha$ is a constant of large value.

This constraint is an additional one to the constraint on the pressure included already, see Eq. (25), and the resulting inf-sup expression is then

$$
\begin{aligned}
& \inf _{q_{h} \in Q_{h}, \lambda_{h} \in A_{h}} \sup _{v_{h} \in V_{h}} \frac{\int_{\mathrm{Vol}} q_{h} \operatorname{div} v_{h} \mathrm{dVol}+\int_{\mathrm{Vol}} \lambda_{h} \operatorname{rot} v_{h} \mathrm{dVol}}{\left\|v_{h}\right\|_{H^{1}}\left(\left\|q_{h}\right\|_{L^{2}}+\left\|\lambda_{h}\right\|_{L^{2}}\right)} \\
& =\gamma_{h} \geqslant \gamma>0 \text {, }
\end{aligned}
$$

where $\Lambda_{h}$ is the discrete space of vorticity moments, $\lambda_{h} \in \Lambda_{h}$, and $\gamma$ is to be a constant independent of $h$. The inf-sup test is performed as described in Section 3.1 and Fig. 2 shows some results obtained in testing acoustic fluid elements [15].

\subsection{Reissner-Mindlin plate bending}

The solution of plate bending problems is accomplished effectively using elements based on ReissnerMindlin plate theory [1]. Let $V_{h}=B_{h} \times W_{h}$ be the finite element interpolation space of the plate section rotations $\beta_{h}$ (in $B_{h}$ ) and transverse displacement $w_{h}$ (in $W_{h}$ ), with $V$ being the space of the continuous problem, and let $G_{h}$ be the finite element interpolation space of the transverse shear strains times $\mu k / t^{2}$. Then the finite element problem is [4]:
Find $u_{h}=\left(\beta_{h}, w_{h}\right) \in V_{h}$ and $\gamma_{h} \in G_{h}=R_{h}\left(V_{h}\right)$ such that ${ }^{1}$

$$
\begin{aligned}
& a^{\mathrm{RM}}\left(\beta_{h}, \eta_{h}\right)+\left(\gamma_{h}, R_{h}\left(\nabla \xi_{h}-\eta_{h}\right)\right)=\left(g, \xi_{h}\right) \\
& \quad \forall v_{h}=\left(\eta_{h}, \xi_{h}\right) \in V_{h},\left(\frac{t^{2}}{\mu k} \gamma_{h}-R_{h}\left(\nabla w_{h}-\beta_{h}\right), \varsigma_{h}\right)=0 \\
& \quad \forall \varsigma_{h} \in G_{h},
\end{aligned}
$$

where $a^{\mathrm{RM}}(\cdot, \cdot)$ is the bilinear form corresponding to the bending action and $g$ represents the transverse load, both normalized by $t^{3}, t$ is the thickness of the plate, $\mu$ is the shear modulus and $k$ is the shear correction factor. The reduction operator $R_{h}$ is introduced to "weaken" the shear strain constraint in order to not have shear locking in the formulation. The operator must be chosen judiciously to preserve consistency and to satisfy the ellipticity and inf-sup conditions $[1,4]$. Note that the formulation in Eq. (36) represents a quite general solution approach.

The inf-sup condition is in this case obtained from Eq. (22) by using the following equivalence for the supremum on the right-hand side:

$c_{1} \sup _{\chi \in \Gamma} \frac{\left(\varsigma_{h}, \chi\right)}{\|\chi\|_{\Gamma}} \geqslant \sup _{v=(\eta, \xi) \in V} \frac{\left(\varsigma_{h}, R_{h}(\nabla \xi-\eta)\right)}{\|v\|_{V}} \geqslant c_{2} \sup _{\chi \in \Gamma} \frac{\left(\varsigma_{h}, \chi\right)}{\|\chi\|_{\Gamma}}$,

where $c_{1}$ are $c_{2}$ are constants and $\Gamma$ corresponds to the continuous space

$\Gamma=\left\{\chi, \chi \in L^{2}(\mathrm{Vol}), \operatorname{rot} \chi \in L^{2}(\mathrm{Vol}), \chi \cdot \tau=0\right.$ on $\left.S\right\}$

with $\tau$ the unit tangent vector on the boundary surface $S$. Hence, the inf-sup condition is [4]

$$
\inf _{\varsigma_{h} \in G_{h}} \sup _{v_{h} \in V_{h}} \frac{\left(\varsigma_{h}, R_{h}\left(\nabla \xi_{h}-\eta_{h}\right)\right)}{\left\|v_{h}\right\|_{V}\left\|\varsigma_{h}\right\|_{\Gamma^{\prime}}}=\gamma_{h} \geqslant \gamma>0
$$

with $\gamma$ independent of $h$, and

$\left\|\varsigma_{h}\right\|_{\Gamma^{\prime}}=\sup _{\chi \in \Gamma} \frac{\left(\varsigma_{h}, \chi\right)}{\|\chi\|_{\Gamma}}$.

The difficulty in evaluating the inf-sup condition lies in that the $\Gamma^{\prime}$-norm involves the complete continuous space. Therefore, the inf-sup test for Eq. (38) consists of two parts. In the first part, the inf-sup expression corresponding to $\Gamma_{h}^{\prime}$ is evaluated.

Consider a given finite element discretization. Using the particular discretization scheme for the transverse shear strains, section rotations and transverse displacement, we have

\footnotetext{
${ }^{1}$ Here $\gamma_{h}$ is the vector of plate bending shear strains times $\mu k / t^{2}$. Since $\gamma_{h} \in G_{h}$ is only used in this equation, a confusion with the inf-sup value used in Eq. (38) is ruled out.
} 


$$
\begin{aligned}
\left\|\chi_{h}\right\|_{\Gamma}^{2} & =\left\|\chi_{h}\right\|_{L^{2}}^{2}+L^{2}\left\|\operatorname{rot} \chi_{h}\right\|_{L^{2}}^{2} \\
& =\boldsymbol{W}_{h}^{\mathrm{T}}\left(\boldsymbol{G}_{h}+\boldsymbol{Q}_{h}\right) \boldsymbol{W}_{h}=\boldsymbol{W}_{h}^{\mathrm{T}} \boldsymbol{D}_{h} \boldsymbol{W}_{h}
\end{aligned}
$$

with $L$ the characteristic length of the plate problem considered, and

$\left\|v_{h}\right\|_{V}^{2}=\left\|\eta_{h}\right\|_{H^{1}}^{2}+\left\|\xi_{h}\right\|_{H^{1}}^{2}=\boldsymbol{V}_{h}^{\mathrm{T}} \boldsymbol{S}_{h} \boldsymbol{V}_{h}$.

In Eqs. (40) and (41), the vectors $\boldsymbol{W}_{h}$ and $\boldsymbol{V}_{h}$ contain the finite element nodal displacement and rotation variables. The inf-sup value $\gamma_{h}$ is given by [4]

$\gamma_{h} \geqslant \gamma_{h}^{*}=\sqrt{\delta_{\min }^{h} / \sigma_{\max }^{h}}$

where $\delta_{\min }^{h}$ stands for the smallest nonzero eigenvalue of $\boldsymbol{D}_{h}$ and $\sigma_{\max }^{h}$ is the largest eigenvalue of $\boldsymbol{S}_{h}$. In practice, the semi-norms instead of the full norms are used in Eq. (41) (based on the Poincaré-Friedrichs inequality [1]).

In the second part, we need to show that the $\Gamma_{h}^{\prime}$ - and $\Gamma^{\prime}$-norms are equivalent so that in fact the conclusions reached using Eq. (38) with the $\Gamma_{h}^{\prime}$-norm are also applicable when the $\Gamma^{\prime}$-norm is used. Considering a sequence of meshes by subdividing the element size $h$ consecutively, we have

$\left\|\varsigma_{h}\right\|_{\Gamma_{h / n}^{\prime}}=\sup _{\chi_{h} \in \Gamma_{h / n}} \frac{\left(\varsigma_{h}, \chi_{h}\right)}{\left\|\chi_{h}\right\|_{\Gamma_{h / n}}} \rightarrow\left\|\varsigma_{h \rightarrow}\right\|_{\Gamma^{\prime}}$.

So that if we consider

$\theta_{n}(h)=\inf _{\varsigma_{h} \in \Gamma_{h}} \frac{\left\|\varsigma_{h}\right\|_{\Gamma_{h}^{\prime}}}{\left\|\varsigma_{h}\right\|_{\Gamma_{h / n}^{\prime}}}$,

we can find an estimate for the constant (when $n$ is large)

$\theta_{\infty}(h)=\inf _{\varsigma_{h} \in \Gamma_{h}} \frac{\left\|\varsigma_{h}\right\|_{\Gamma_{h}^{\prime}}}{\left\|\varsigma_{h}\right\|_{\Gamma^{\prime}}}$.

Then if

$\theta_{\infty}(h) \geqslant \theta>0$

for some $\theta$ independent of $h$, we can conclude that the $\Gamma_{h^{\prime}}^{\prime}$ and $\Gamma^{\prime}$-norms are equivalent. In some cases, it is possible to prove analytically that $\theta_{2}(h)$ is a lower bound on $\theta_{\infty}(h)$ [16] but a general numerical procedure is also given in Refs. $[4,16]$.

An alternative way to proceed is to consider a plate element to be a special case of a shell element and use the inf-sup test considered in the next section.

We should note that the formulation given in Eq. (36) is quite general, but the effectiveness of a discretization depends of course on the specific interpolation spaces chosen and the reduction operator $R_{h}$. The choice of the function spaces for the MITC family of plate bending elements, which satisfy the consistency, ellipticity and inf-sup conditions, is discussed in Refs. $[1,17]$ and Fig. 3 shows some inf-sup test results for a few

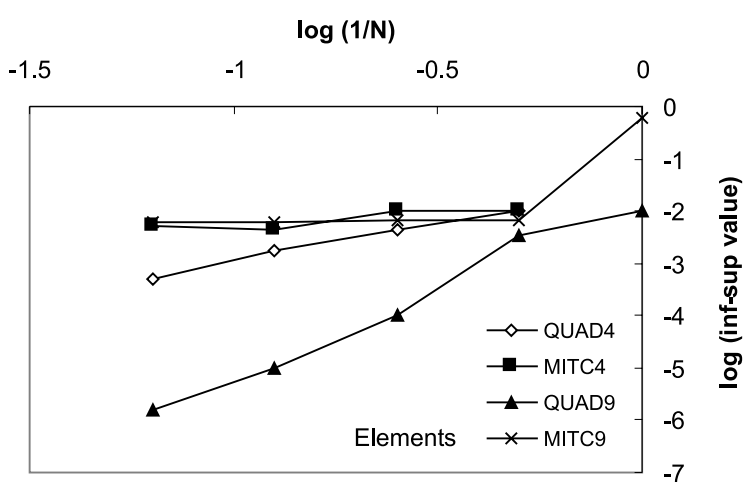

Fig. 3. Inf-sup test results for some MITC plate-bending elements. A clamped square plate using meshes of $N$ by $N$ distorted elements is considered [4].

MITC plate bending elements. (Note that the values of $\gamma_{h}^{*}$, see Eq. (42), are plotted here).

\subsection{General shell conditions}

An effective shell finite element must be applicable to membrane- and bending-dominated shell problems. Ideally, the element shows optimal convergence characteristics in both problem areas. Elements formulated using pure displacement interpolations are effective in membrane-dominated situations but "lock" when bending is encountered. To arrive at a general shell finite element discretization, a mixed formulation must be used. The formulation must preserve consistency for all strain terms, satisfy the ellipticity condition and, considering the bending-dominated case, satisfy the inf-sup condition.

For the analysis of general shell problems, the MITC shell elements are quite effective $[1,18]$. Let $\mathscr{U}_{h}$ be the displacement interpolation space (including the displacements and section rotations) and $\mathscr{E}_{h}$ be the strain interpolation space; then the MITC shell element formulation considering the bending-dominated case can be written in the classical form of Eq. (16):

Find $U_{h} \in \mathscr{U}_{h}$ and $E_{h} \in \mathscr{E}_{h}$ such that

$$
\begin{aligned}
& A\left(\varepsilon^{\mathrm{AS}}\left(U_{h}\right), \varepsilon^{\mathrm{AS}}\left(V_{h}\right)\right)+B\left(E_{h}, V_{h}\right)=\left(F_{b}, V_{h}\right) \quad \forall V_{h} \in \mathscr{U}_{h}, \\
& B\left(\psi_{h}, U_{h}\right)-t^{2} C\left(E_{h}, \psi_{h}\right)=0 \quad \forall \psi_{h} \in \mathscr{E}_{h},
\end{aligned}
$$

where $A(\cdot, \cdot)$ is the bilinear form containing the bending strains $\varepsilon^{\mathrm{AS}}$ obtained by tying to the displacements, $E_{h}$ corresponds to the membrane and shear strain components divided by $t^{2}, t$ is the shell thickness, $B(\cdot, \cdot)$ is the bilinear form corresponding to the membrane and shear actions, $C(\cdot, \cdot)$ is the bilinear form constraining with $B(\cdot, \cdot)$ the strain interpolations to the displacements, and $\left(F_{b}, \cdot\right)$ is the linear form of the loads divided by $t^{3}$. Since 
the loading is scaled by $t^{3}$, the bending-dominated case is considered.

The inf-sup condition employed is [5]

$\inf _{\psi_{h} \in \mathscr{E}_{h}} \sup _{V_{h} \in \mathscr{U}_{h}} \frac{B\left(\psi_{h}, V_{h}\right)}{\left\|V_{h}\right\|_{\mathscr{U}}\left\|\psi_{h}\right\|_{L^{2}}}=\gamma_{h} \geqslant \gamma>0$,

where we used that

$B(\psi, V) \leqslant c\|\psi\|_{L^{2}}\|V\|_{\mathscr{U}} \quad \forall(\psi, V) \in L^{2} \times \mathscr{U}$

and hence for Eq. (22)

$\sup _{V \in \mathscr{U}} \frac{B(\psi, V)}{\|V\|_{\mathscr{U}}} \leqslant c\|\psi\|_{L^{2}}$,

where $c$ is a constant.

The bilinear form $B(\cdot, \cdot)$ evaluates the membrane and shear strain energies and these can be calculated introducing

$\boldsymbol{W}_{h}^{\mathrm{T}} \widetilde{\boldsymbol{K}}_{h} \boldsymbol{V}_{h}=C\left(\varepsilon^{\mathrm{AS}}\left(W_{h}\right), \varepsilon^{\mathrm{AS}}\left(V_{h}\right)\right)$,

where $\widetilde{\boldsymbol{K}}_{h}$ is in practice obtained by simply integrating the usual strain terms but only on the mid-surface of the shell (that is, using one-point integration in the element thickness direction).

The matrix $S_{h}$ is used to calculate the norm on $V_{h}$,

$\left\|V_{h}\right\|_{\mathscr{U}}^{2}=\boldsymbol{V}_{h}^{\mathrm{T}} \boldsymbol{S}_{h} \boldsymbol{V}_{h}$.

Instead of the $L^{2}$-norm on $\psi_{h}$, we use the equivalent expression $\sqrt{C\left(\psi_{h}, \psi_{h}\right)}$, and then the inf-sup value $\gamma_{h}$ in Eq. (48) can be evaluated by calculating the smallest nonzero eigenvalue $\lambda_{k}$ of the problem

$\widetilde{\boldsymbol{K}}_{h} \boldsymbol{\phi}=\lambda \boldsymbol{S}_{h} \boldsymbol{\phi}$.

The value $\sqrt{\lambda_{k}}$ is the inf-sup value $\gamma_{h}$ in Eq. (48) for the discretization considered. The inf-sup test is performed as described in Section 3.1. In the test, of course, a bending-dominated problem needs to be considered, and according to our experience a hyperbolic paraboloid shell provides a suitable problem [5]. This same test can of course also be applied to Reissner-Mindlin plate bending elements and to Timoshenko beam elements. If the test is passed, the element is effective for bendingdominated problems. However, if the test is not passed, we cannot conclude that the element does not satisfy the inf-sup condition in Eq. (22) because the conditions in Eqs. (22) and (48) are not equivalent (see Eq. (50)).

Fig. 4 shows some inf-sup test results calculated for MITC shell elements [5].

\subsection{High Péclet number flow}

Consider a high Péclet number flow problem, in which the velocity is prescribed so that the only unknown is the temperature, $\theta$. The finite element problem is [1]: (a)

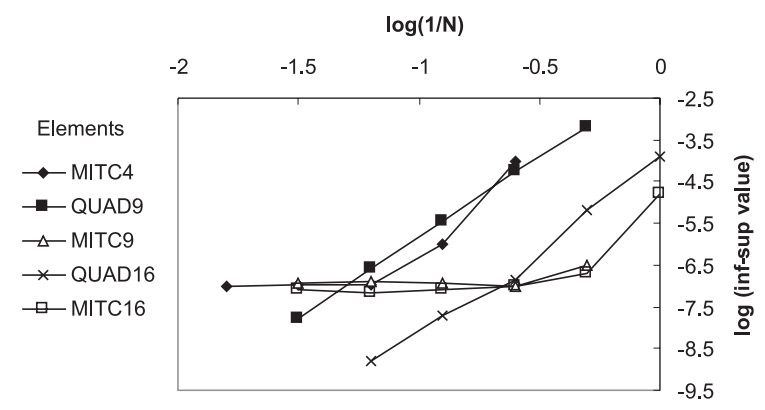

(b)

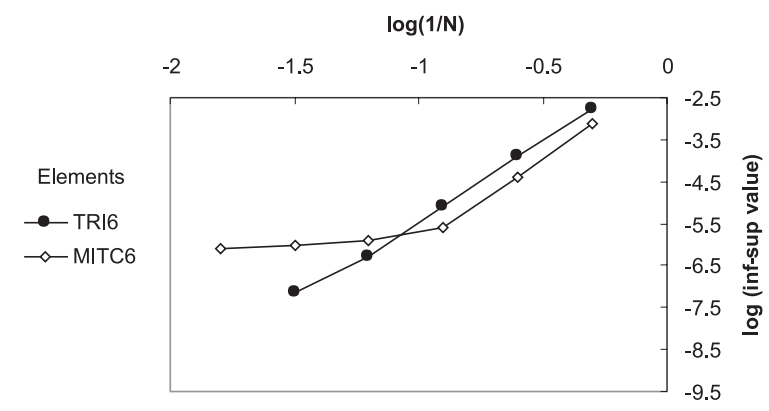

Fig. 4. Inf-sup test results for MITC shell elements. A partly clamped hyperbolic paraboloid shell using uniform meshes is considered [5]: (a) Quadrilateral elements and (b) triangular elements.

Find $\theta_{h} \in \mathscr{U}_{h}$ such that

$a\left(\theta_{h}, \psi_{h}\right)=\left(b, \psi_{h}\right) \quad \forall \psi_{h} \in \boldsymbol{U}_{h}$.

This problem is of the kind discussed in Section 2.1, where we note that the bilinear form $a(\cdot, \cdot)$ is nonsymmetric. The inf-sup condition was given as

$\inf _{\xi_{h} \in \mathscr{U}_{h}} \sup _{\psi_{h} \in \mathscr{U}_{h}} \frac{a\left(\xi_{h}, \psi_{h}\right)}{\left\|\xi_{h}\right\|_{S}\left\|\psi_{h}\right\|_{T}}=\gamma_{h} \geqslant \gamma>0$.

For the evaluation of the inf-sup value we define the matrices $\boldsymbol{A}_{h}, \boldsymbol{S}_{h}$ and $\boldsymbol{T}_{h}$. Considering a given discretization we have, using Eq. (54) [6]

$\boldsymbol{A}_{h} \boldsymbol{x}=\boldsymbol{b}$

and the norms

$\left\|\xi_{h}\right\|_{S}^{2}=\boldsymbol{\xi}_{h}^{\mathrm{T}} \boldsymbol{S}_{h} \boldsymbol{\xi}_{h}$,

$\left\|\psi_{h}\right\|_{T}^{2}=\boldsymbol{\psi}_{h}^{\mathrm{T}} \boldsymbol{T}_{h} \boldsymbol{\psi}_{h}$

where $\xi_{h}$ and $\psi_{h}$ are the nodal values corresponding to $\xi_{h}$ and $\psi_{h}$, respectively. Then, as derived in Ref. [6], the infsup value $\gamma_{h}$ is obtained by solving the following eigenvalue problem for the smallest eigenvalue $\lambda_{\min }$, 


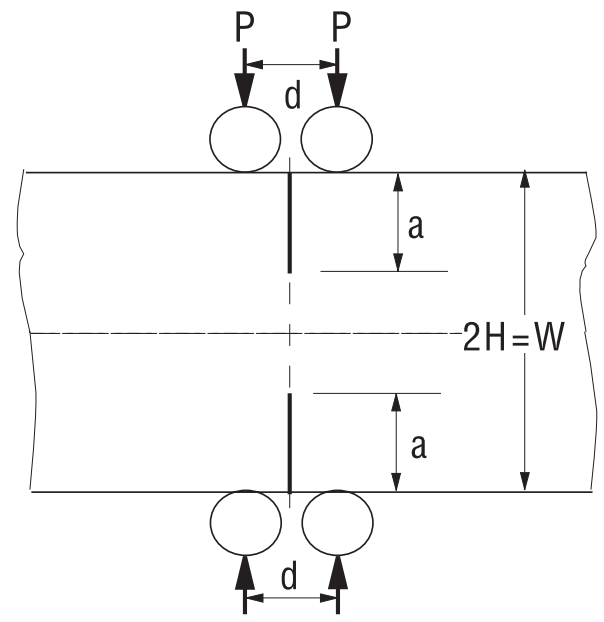

Fig. 5. Inf-sup test results for one-dimensional high Péclet number flow problem [6]. The convection-diffusion problem corresponds to a constant prescribed velocity throughout the domain and prescribed temperatures at both ends of the domain. 16 equal-sized elements are used.

$\left(\boldsymbol{A}_{h}^{\mathrm{T}} \boldsymbol{T}_{h}^{-1} \boldsymbol{A}_{h}\right) \boldsymbol{\phi}=\lambda \boldsymbol{S}_{h} \boldsymbol{\phi}$.

The inf-sup value $\gamma_{h}$ is equal to $\sqrt{\lambda_{\min }}$ and in general depends on the Péclet number (Pe). The inf-sup test is performed by calculating $\gamma_{h}$ for a given discretization as the Péclet number is increased to very large values. If the inf-sup value does not decrease, but stabilizes to remain larger than a value greater than zero, the test is passed.

Alternatively, we can consider a problem of very high Péclet number, use a fine discretization and then calculate $\gamma_{h}$ for increasingly coarser meshes. The test is passed if $\gamma_{h}$ does not decrease but stabilizes above a value larger than zero.

These tests were performed in Ref. [6] for various upwind schemes applied to a one-dimensional test problem (Fig. 5). The curves given in Fig. 5 show that, as expected, the standard Galerkin method is unstable whereas the other methods are stable. The inf-sup test results also show the amount of artificial diffusion contained in the method; that is, the smaller the inf-sup values, the less artificial diffusion is contained in the method.

\section{Concluding remarks}

The objective of this paper was to present some latest experiences in the use and evaluation of the inf-sup condition for mixed finite element formulations. This condition is frequently the crucial condition to ensure the stability and optimality of a discretization scheme. However, it can be difficult, or impossible, to evaluate analytically whether the condition is satisfied. For this reason, numerical inf-sup tests have been designed. As shown in this paper these tests are applied in a straightforward manner (like the patch test for displacementbased incompatible models) to discretizations applicable to various analysis fields. Inf-sup tests for analysis fields not considered herein can be similarly performed.

Our experiences are that when the numerical inf-sup test is passed, the inf-sup condition is satisfied. Hence, mixed finite element formulations can now be evaluated as shown in this paper before it is claimed that an effective formulation has been developed.

\section{Acknowledgements}

I would like to thank F. Brezzi, University of Pavia and D. Chapelle, INRIA for their long and fruitful cooperation with me on the theory and methods discussed in this paper.

\section{References}

[1] Bathe KJ. Finite element procedures. Englewood Cliffs, NJ: Prentice Hall; 1996.

[2] Brezzi F, Bathe KJ. A discourse on the stability conditions for mixed finite element formulations. Comp Meth Appl Mech Engng 1990;82:27-57.

[3] Chapelle D, Bathe KJ. The inf-sup test. Comput Struct 1993;47:537-45.

[4] Iosilevich A, Bathe KJ, Brezzi F. On evaluating the inf-sup condition for plate bending elements. Int J Num Meth Engng 1997;40:3639-63.

[5] Bathe KJ, Iosilevich A, Chapelle D. An inf-sup test for shell finite elements. Comput Struct 2000;75:439-56.

[6] Bathe KJ, Hendriana D, Brezzi F, Sangalli G. Inf-sup testing of upwind methods. Int $\mathrm{J}$ Num Meth Engng 2000;48:745-60.

[7] Brezzi F, Pitkäranta J. On the stabilization of finite element approximations of the Stokes equations. In: Hackbush W, editor, Efficient solutions of elliptic systems. Notes on numerical fluid mechanics, vol. 10, p. 11-9. Vieweg Braunschweig, Wiesbaden, 1984.

[8] Hughes TJR, Franca LP, Balestre M. A new finite element formulation for computational fluid dynamics: V. Circumventing the Babuška-Brezzi condition: A stable Petrov-Galerkin formulation of the Stokes problem accommodating equal-order interpolations. Comp Meth Appl Mech Engng 1986;59:85-99.

[9] Banach S. Théorie des Opérations Linéaires. Warszawa, 1932.

[10] Babuška I. The finite element method with Lagrangian multipliers. Numerische Mathematik 1973;20:179-92.

[11] Brezzi F. On the existence, uniqueness and approximation of saddle-point problems arising from Lagrangian multipliers. Revue française d'automatique informatique recherche operationnelle. Analyse Numérique 1974;8:129-51.

[12] Ladyzhenskaya OA. The mathematical theory of viscous incompressible flow. New York: Gordon and Breach; 1969. 
[13] Pantuso D, Bathe KJ. A four-node quadrilateral mixedinterpolated element for solids and fluids. Math Models Meth Appl Sci 1995;5:1113-28.

[14] Wang X, Bathe KJ. Displacement/pressure based mixed finite element formulations for acoustic fluid-structure interaction problems. Int J Num Meth Engng 1997;40:2001-17.

[15] Wang X, Bathe KJ. On mixed elements for acoustic fluidstructure interactions. Math Models Meth Appl Sci 1997; 7:329-43.
[16] Iosilevich A, Bathe KJ, Brezzi F. Numerical inf-sup analysis of MITC plate bending elements. Proceedings, 1996 American Mathematical Society Seminar on Plates and Shells. Université Laval, Canada, 1996.

[17] Bathe KJ, Bucalem ML, Brezzi F. Displacement and stress convergence of the MITC plate bending elements. J Engng Computat 1990;7:291-302.

[18] Bathe KJ, Iosilevich A, Chapelle D. An evaluation of the MITC shell elements. Comput Struct 2000;75:1-30. 


\title{
Erratum
}

\section{Erratum to "The inf-sup condition and its evaluation for mixed finite element methods" \\ [Computers and Structures 79 (2001) 243-252] is}

\author{
Klaus-Jürgen Bathe * \\ Department of Mechanical Engineering, Massachusetts Institute of Technology, 77 Mass Avenue, Cambridge, MA 02139, USA
}

The Publisher regrets that Fig. 5 in the printed version of the above article is incorrect. The correct figure appears below. We apologise for any inconvenience and concerns caused by this matter.

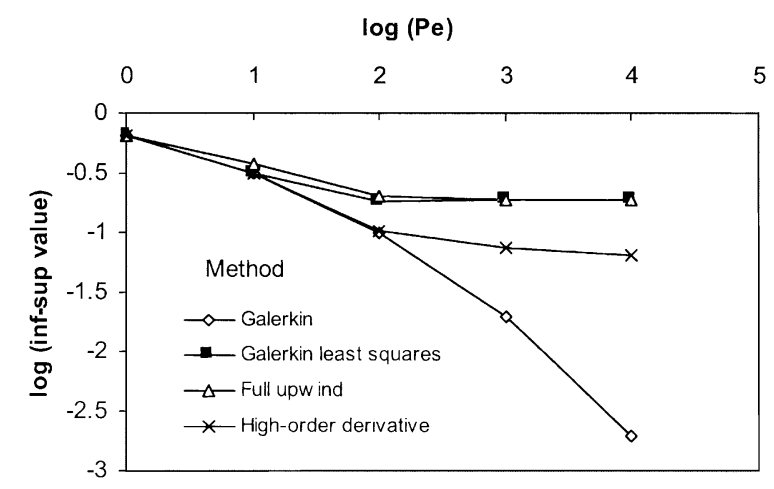

Fig. 5. Inf-sup test results for one-dimensional high Péclet number flow problem [6]. The convection-diffusion problem corresponds to a constant prescribed velocity throughout the domain and prescribed temperatures at both ends of the domain. 16 equal-sized elements are used.

\footnotetext{
PII of original article: S0045-7949(00)00123-1.

*Tel.: +1-617-2536645; fax: +1-617-2532275.

E-mail address: kjb@mit.edu (K.J. Bathe).
} 